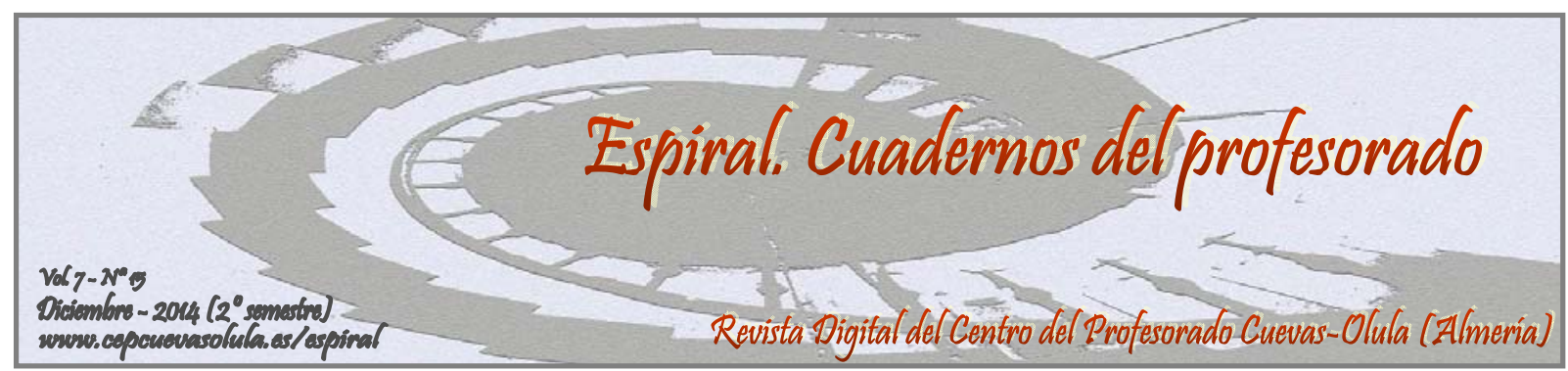

\title{
EL TRIATLÓN: UNA PROPUESTA DE ACTIVIDAD FISICA EN EL MEDIO NATURAL
}

\section{TRIATHLON: A PROPOSAL OF PHYSICAL ACTIVITY IN THE NATURAL ENVIRONMENT}

\author{
Victoria Eugenia Machota Blas
}

IES D. Antonio Hellín Costa (Puerto de Mazarrón, Murcia)

RESUMEN: Esta comunicación presenta una propuesta innovadora mediante la implementación del deporte de Triatlón en un contexto de educación física escolar con objeto de fomentar la actividad física en el medio natural para mantenerse activo, saludable y en forma. La unidad se compone de 12 lecciones llevadas a cabo en un centro escolar situado en un municipio costero de Murcia. Los estudiantes trabajaron las técnicas básicas de cada una de las tres disciplinas y las transiciones. Los resultados sobre el impacto de Triatlón en los estudiantes fueron estudiados a través de grupos de discusión. Los alumnos parecen haber tomado conciencia del deporte del triatlón y demostraron resultados sobre el conocimiento, la comprensión y las habilidades relacionadas con este deporte. Esta unidad ha proporcionado a los adolescentes las oportunidades de aprender estas habilidades y ha supuesto un acercamiento de los alumnos a las actividades físicas en la naturaleza despertando su interés por este medio y hacia el deporte del triatlón (triatlón como un estilo de vida). Su desarrollo inicial puede parecer condicionada a la ubicación y características de material muy específico, pero con las modificaciones apropiadas puede ser adaptado perfectamente e incluido en la programación de la educación física en las escuelas.

Palabras clave: Actividad física, medio natural, triatlón, unidad didáctica, educación secundaria obligatoria.

ABSTRACT: This paper presents an innovative approach and implementation of Triathlon in a school-based Physical Education context in order to promote outdoor physical activity. A teaching unit of triathlon was designed to become part of the Physical Education syllabus. It introduced adolescents to Triathlon fostering physical activity in the natural environment to stay active, healthy and fit. The unit was made up of 12 lessons conducted in a Secondary School situated on the southeast coast of Spain (Murcia). Students worked on the basic techniques of each of the three disciplines and the transitions. The results on the impact of Triathlon on students were studied through discussions with focus groups. Students seem to have become aware of the sport of Triathlon and demonstrate outcomes on knowledge, understanding and basic skills related to Triathlon. This unit has provided adolescents the opportunities to learn these skills and practise this sport beyond school based Physical Education (triathlons as a lifestyle choice) and increase their levels of physical activity. Its initial development may seem conditioned upon location and characteristics of very specific material, but with appropriate modifications it can be adapted perfectly and included in the school-based Physical Education syllabus.

Key words: triathlon, physical education, teaching unit, outdoor education. 
Machota Blas, V. E. (2014). El triatlón: una propuesta de actividad física en el medio natural. Espiral. Cuadernos del Profesorado, 7(15), 28-35. Disponible en: http://www.cepcuevasolula.es/espiral.

Fecha de recepción: 08/05/2014

Fecha de aceptación: 27/09/2014
Enviar correspondencia a: vmictoriaeugen6@gmail.com

\section{1.- INTRODUCCIÓN}

Tierra Orta (1996) define las actividades en el medio natural como un conjunto de actividades de carácter interdisciplinar que se desarrollan en contacto con la naturaleza, con finalidad educativa, recreativa y deportiva, y con cierto grado de incertidumbre en el medio. A partir de este concepto, el triatlón es una actividad que tiene lugar en contacto con el medio natural y se define como la combinación de natación, ciclismo y carrera a pie realizados en este orden, y sin descanso entre uno y otro, en el cuál el segmento de natación se desarrolla en entorno natural de aguas abiertas (lagos, pantanos, playas, ríos,...).

Tradicionalmente se han clasificado las actividades en la naturaleza según el medio físico implicado en su desarrollo (Granero, 2007):

- Actividades de tierra: bicicleta de montaña, marcha ecuestre, trekking, marchas, campo a través, acampada, montañismo, escalada, espeleología, carrera de orientación, turismo ecuestre...

- Actividades de aire: parapente, puenting, benjing, ala delta, paracaidismo, vuelo sin motor, aerostación...

- Acuáticas: natación, waterpolo, natación sincronizada, socorrismo, natación con aletas, descenso de barrancos...

- Actividades del mundo animal: pesca, caza, equitación.

El triatlón es una actividad física en el medio natural que se ubica en el grupo de actividades acuáticas y de actividades de tierra cuando se realiza con una bicicleta de montaña y/ o la carrera a pie se desarrolla en un entorno natural. En los últimos tiempos, las modalidades de este tipo (duatlón cross y triatlón cross) han experimentado gran un auge en cuanto a participación y número de competiciones y la tendencia cada vez más es a buscar circuitos en entornos naturales (circuito europeo de triatlón cross “TNATURA”, circuito "Xterra”, etc.).

En los años recientes, el triatlón se ha convertido en un deporte muy popular en España y en todo el mundo experimentando un notable incremento en su participación y práctica. El Consejo Superior de Deportes (CSD) en colaboración con la Federación Española de Triatlón (FETRI) y las federaciones territoriales están promoviendo su práctica y para ello se ha modificado el reglamento y las distancias adaptando este deporte a todos los niveles, en especial a los más pequeños y jóvenes.

El triatlón presenta diferentes combinaciones que darán lugar a las distintas modalidades y, a su vez, presenta distancias variadas. Existen modalidades de triatlón (natación, bicicleta y carrera a pie), duatlón (carrera a pie, bicicleta y carrera a pie) y acuatlón (carrera a pie, natación y carrera a pie). También existe el llamado triatlón cross y duatlón cross que se diferencian por utilizar bicicleta de montaña y transcurrir por circuitos de tierra. Además de las modalidades anteriores, existe el triatlón de invierno que combina carrera a pie, ciclismo y esquí y, el quatriatlón con segmentos de piragua, natación, ciclismo y carrera. A continuación detallamos las distancias de cada uno de los segmentos según las modalidades más conocidas (Torres y Portoles, 2007) (Tabla 1).

El triatlón es uno de los deportes incluidos en el programa de Deporte Escolar que desarrollan el CSD y las Comunidades Autónomas. De este modo, existen competiciones en categorías inferiores en las que pueden participar los alumnos cuyo fin es el aumento de la práctica deportiva. A ello se une su inclusión en el Programa Nacional de Promoción y Ayuda al Deporte Escolar (PROADES) 
desarrollado también por el CSD, la FETRI y las federaciones territoriales. Las distancias para categorías menores son más cortas y respetuosas con el desarrollo infantil y existe un reglamento para las categorías en edad escolar con las siguientes opciones recomendadas (Torres y Portoles, 2007) (Tabla 2).

Tabla 1. Distancias (metros) de cada uno de los segmentos según las modalidades.

\begin{tabular}{cccc}
\hline TRIATLÓN & Natación & Ciclismo & Carrera \\
\hline Sprint & 750 & 20.000 & 5.000 \\
Olímpica & 1.500 & 40.000 & 10.000 \\
\hline TRIATLÓN CROSS & Natación & Ciclismo & Carrera \\
\hline Sprint & 750 & 10.000 & 4.000 \\
\hline DUATLÓN & Carrera & Ciclismo & Carrera \\
\hline Sprint & 5.000 & 20.000 & 2.500 \\
Corta & 10.000 & 40.000 & 5.000 \\
Larga & 20.000 & 80.000 & 10.000 \\
\hline DUATLÓN CROSS & Carrera & Ciclismo & Carrera \\
\hline Sprint & 4.000 & 10.000 & 2.000 \\
\hline ACUATLÓN & Carrera & Natación & Carrera \\
\hline Sprint & 2.500 & 1.000 & 2.500 \\
Corta & 5.000 & 2.000 & 5.000 \\
\hline
\end{tabular}

Por último, la promoción a través de la campaña "SERTRI" (Cadena Ser y la FETRI) pretenden difundir la práctica de este deporte y fomentar la iniciación en edades tempranas. Para ello se vienen celebrando competiciones por distintos puntos de España y se ha creado el triatlón "flash" $(150 \mathrm{~m}-4 \mathrm{~km}-1 \mathrm{~km})$ y otras posibles combinaciones y distancias.

Tabla 2. Distancias (metros) categorías menores

\begin{tabular}{cccc}
\hline TRIATLÓN & Natación & Ciclismo & Carrera \\
\hline Prebenjamín & 50 & 1.000 & 250 \\
Benjamín & 100 & 2.000 & 500 \\
Alevín & 200 & 4.000 & 1.000 \\
Infantil & 400 & 8.000 & 2.000 \\
\hline DUATLÓN & Carrera & Ciclismo & Carrera \\
\hline Prebenjamín & 250 & 1.000 & 125 \\
Benjamín & 500 & 2.000 & 250 \\
Alevín & 1.000 & 4.000 & 500 \\
Infantil & 2.000 & 8.000 & 1.000 \\
\hline ACUATLÓN & Carrera & Natación & Carrera \\
\hline Prebenjamín & 125 & 50 & 125 \\
Benjamín & 250 & 100 & 250 \\
Alevín & 500 & 200 & 500 \\
Infantil & 1.000 & 400 & 1.000 \\
\hline
\end{tabular}




\section{2.- PROPUESTA DE UNIDAD DIDÁCTICA}

\section{Contextualización}

Esta unidad didáctica (UD) (Machota, 2012) esta diseñada y ha sido desarrollada atendiendo a las características del entorno socio-ambiental de un centro concreto y considerando los conocimientos de la docente de Educación Física, autora aficionada a este deporte. El centro en cuestión es el Instituto de Enseñanza Secundaria "Antonio Hellín Costa” situado en el Puerto de Mazarrón (sureste de la Región de Murcia) que se encuentra ubicado en una zona costera con un entorno y clima ideal para la práctica del triatlón. No obstante, la singularidad de cada centro de enseñanza permite las adaptaciones necesarias a la hora de organizar y desarrollar una UD de triatlón.

La UD esta diseñada bajo el marco del Proyecto Educativo del Centro y se trata de una actividad contemplada en la Programación didáctica del departamento de Educación Física para los alumnos de $3^{\circ}$ y $4^{\circ}$ de ESO. Se viene desarrollando en el tercer trimestre atendiendo a razones de clima con una temporalización de ocho sesiones en el propio centro y dos salidas complementarias. Estas salidas se desarrollarán en el propio medio natural: una salida a la playa para la realización de un acuatlón y otra salida para realizar un recorrido en bicicleta por el entorno natural próximo. En ambos casos los alumnos salen del centro con la correspondiente autorización de los padres y se dedica toda la jornada escolar a las mismas. Por otra parte, la UD se enriquece con la participación de los alumnos en las competiciones de duatlón, acuatlón y triatlón que se celebran dentro del programa de Deporte Escolar desarrollado por la Comunidad Autónoma de la Región de Murcia y la posibilidad de participar en el circuito de competiciones regional desarrollado por la Federación de Triatlón de la Región de Murcia (FTRM).

\section{Competencias básicas desarrolladas}

Las competencias básicas hacen referencia a aquellos aprendizajes que se consideran imprescindibles para capacitar a los alumnos en su realización personal, el ejercicio de la ciudadanía activa, la incorporación satisfactoria a la vida adulta y el desarrollo de un aprendizaje permanente a lo largo de la vida (Real Decreto 1631/2006). Cada una de las áreas debe contribuir al desarrollo de diferentes competencias. Esta UD contribuirá a la adquisición de las siguientes competencias:

- Competencia en comunicación lingüística: La UD ofrece una variedad de intercambios comunicativos con un vocabulario específico y palabras clave en el mundo del triatlón.

- Competencia en el conocimiento e interacción con el mundo: las actividades propuestas en la naturaleza son un medio de consolidar hábitos y actitudes personales de interrelación con el medio; proporcionan conocimientos y destrezas sobre determinados hábitos saludables que pretenden acompañar a los alumnos más allá de la etapa obligatoria y requieren de la participación e implicación activa de la persona.

- Tratamiento de la información y competencia digital: los alumnos aprenden a manejar información en diferentes medios acerca del triatlón.

- Competencia social y ciudadana: Las salidas para realizar actividades físicas en la naturaleza son un medio eficaz para facilitar la socialización y convivencia en común, la integración y el respeto, a la vez que contribuyen al desarrollo de la cooperación y la solidaridad. A ello se une el respeto a las normas y reglamento del triatlón.

- Competencia para aprender a aprender: las actividades propuestas favorecen la autonomía e independencia y permiten que el alumnado sea capaz de regular su propio aprendizaje y práctica de la actividad física en su tiempo libre, de forma organizada y estructurada.

- Autonomía e iniciativa personal: Las actividades en la naturaleza suponen el enfrentamiento, vivencia y superación de situaciones de riesgo que lleva consigo una autoafirmación personal, autocontrol personal y autogestión de la motricidad (Ascaso Martorell, Casterad Seral, Generelo Lanaspa, Guillén Correas, Lapetra Costa, Tierz Garcia, 1996). En la UD se otorga protagonismo al alumnado enfrentándole a situaciones en las que debe mani- 
festar autosuperación, perseverancia, responsabilidad y actitud positiva. Se refuerza el conocimiento de las propias posibilidades y limitaciones valorando las diferencias individuales debido a las características de esfuerzo y superación que fomentan el deporte del triatlón.

\section{Objetivos}

Teniendo presente las competencias básicas, la UD planteada pretende contribuir a alcanzar los Objetivos Generales de la Etapa de la ESO (Real Decreto 1631/2006) y del área de Educación Física para esta etapa (Decreto no 291/2007) recogidos en el Proyecto Educativo del Centro. A la luz de estos, se determinan los siguientes objetivos didácticos:

- Conocer y vivenciar las posibilidades de práctica de triatlón en el medio natural y sus modalidades.

- Practicar actividad física en el medio natural respetándolo y conservándolo.

- Realizar un calentamiento específico para el triatlón, duatlón y acuatlón.

- Participar y colaborar en un triatlón, duatlón y/o acuatlón independientemente del nivel de destreza alcanzado.

- Conocer y respetar las normas de circulación vial y los riesgos de la práctica de actividad física en el medio natural.

- Mejorar las habilidades específicas del ciclismo y promover el uso de la bicicleta como medio de transporte autónomo no contaminante.

- Valorar positivamente la práctica de triatlón como medio recreativo, de ocupación del tiempo libre y de desarrollo y mantenimiento de la salud.

\section{Contenidos}

La UD se relaciona con el bloque de contenidos de actividades en el medio natural. Paralelamente también tiene relación con los bloques de juegos y deportes; cualidades motrices personales y, condición física y salud (Decreto n ${ }^{\circ}$ 291/2007). A partir de esta primera aproximación, definimos los siguientes contenidos específicos:

\section{Conceptos:}

- Historia, características, modalidades y reglamento del triatlón.

- Los segmentos y transiciones del triatlón.

- La bicicleta en el triatlón y como medio de transporte (educación vial).

Procedimientos:

- Calentamiento específico de triatlón, duatlón y acuatlón.

- Técnica de los diferentes segmentos: natación, bicicleta y carrera a pie.

- Técnica de las transiciones en triatlón.

- Manejo, uso y mantenimiento básico de la bicicleta.

Actitudes

- Valoración positiva de la práctica de triatlón como medio recreativo, de ocupación del tiempo libre y de desarrollo y mantenimiento de la salud.

- Interiorización de la importancia del entorno natural como medio de disfrute y ocio.

- Respeto de las normas y seguridad en la circulación vial y uso de la bicicleta como medio de transporte saludable y no contaminante.

- Respeto al medio natural y cuidado del mismo.

- Participación activa en el deporte de triatlón, independientemente del nivel de destreza alcanzado conociendo las limitaciones de cada uno. 


\section{Metodología}

La metodología será activa y participativa. Se trata de crear un ambiente motivante que despierte el deseo de práctica de este deporte. Se procurará una pedagogía no directiva planteando situaciones para la exploración de diversas prácticas sobre todo en las sesiones dedicadas a la transiciones y bicicleta, aprendizajes por descubrimiento o búsqueda a partir de consignas abiertas. Los alumnos trabajarán en parejas y grupos a modo de relevos con objeto de alcanzar objetivos comunes. Para la competición que se celebra en la playa los alumnos competirán como un equipo cabiendo dos posibilidades diferentes: todos los miembros del equipo realizan todos los segmentos o cada segmento es realizado por un solo miembro del equipo.

\section{Recursos}

- Recursos ambientales: las salidas complementarias de la UD se desarrollan en el entorno natural próximo al centro. Se ha elegido la playa más próxima al centro y la que mayor seguridad y menor riesgo ofrece tratándose de la playa de "Bahía”. El desplazamiento a esta playa se ha realizado caminando aproximadamente unos $2 \mathrm{~km}$. La salida en bicicleta se ha realizado por pistas de tierra en zona próxima al centro y en colaboración con la policía local del municipio.

- Recursos materiales: el triatlón es un deporte que requiere de diverso material que en esta UD se ha reducido al mínimo. Para las dos primeras sesiones teóricas se han utilizado las nuevas tecnologías con presentaciones en "powerpoint”, el uso de diferentes páginas web (www.trimurcia.org, www.triatlón.org, etc.), videotutoriales y de competiciones así como el programa "Guía” ofrecido por la Federación Española de Triatlón (http://tecnificacion.triatlon.org/programa-guía). Para las sesiones prácticas con bicicleta se han utilizado las bicicletas de los alumnos admitiendo todo tipo de bicicletas .y los cascos de los mismos. En esos días aquellos alumnos con bicicleta y con la posibilidad de desplazarse al centro en ella así lo han hecho. De este modo, las bicicletas aportadas se han utilizado en los diferentes grupos organizando a los alumnos en grupos para realizar las prácticas. El material para reparación de la bicicleta (cámaras, parches, herramientas, etc.) lo ha aportado el centro. Para la salida complementaria de realización de un recorrido en bicicleta los alumnos también han utilizado la suya (siempre con casco) y han sido avisados con tiempo para ponerla a punto y, en caso de no disponer de una bicicleta, conseguir alguna prestada. Para la realización de las demás prácticas y la competición en la playa se han empleado dorsales, gomas elásticas para su colocación, gorros de natación, conos y picas, material aportado por el centro. Las sesiones prácticas de bicicleta, carrera y transiciones se han desarrollado en una pista polideportiva exterior.

\section{Actividades de enseñanza-aprendizaje}

A continuación se expone el desarrollo de las sesiones de esta UD con los objetivos y actividades principales de cada una de ellas:

Sesión 1 -TRIATLÓN

- Introducción: Historia, características, modalidades y transiciones.

- Reglamento y material del triatleta.

Sesión 2-EL SEGMENTO DE NATACIÓN

- Características del segmento de natación en triatlón: el estilo crol.

- Natación en aguas abiertas: normas de seguridad y reglamento.

Sesión 3- LA BICICLETA

- La bicicleta.

- Normas de Circulación.

- Descripción Técnica de las acciones básicas. 
- Reparaciones Básicas.

Sesión 4- SEGMENTO DE BICICLETA

- El ciclismo en triatlón y sus normas.

- Ejercicios y circuito de habilidad con la bicicleta.

Sesión 5- SEGMENTO DE CARRERA

- La carrera a pie en triatlón.

- Ejercicios y práctica de carrera.

Sesión 6- TRANSICIONES

- Técnica de las transiciones.

- Ejercicios y práctica de transiciones.

- Sesión 7 - TRANSICIONES

- Práctica de transiciones y ejecución prueba práctica.

Sesión 8 - EXAMEN TEÓRICO

- Examen teórico sobre los contenidos y procedimientos desarrollados.

- Respuesta anónima de los alumnos a una hoja de evaluación de la UD desarrollada.

Actividad complementaria: Competición en la playa: acuatlón/triatlón.

Actividad complementaria: Salida en bicicleta por el entorno natural próximo.

\section{Evaluación}

A partir de los objetivos didácticos y en función de los contenidos específicos, se definen los siguientes indicadores de evaluación y porcentajes de calificación:

Tabla 3. Indicadores y \% calificación

\begin{tabular}{llc}
\hline Indicadores & $\%$ \\
\hline - & $\begin{array}{l}\text { Identifica los orígenes y actualidad del triatlón, sus modalidades y distancias y normas y regla- } \\
\text { mento básico }\end{array}$ & $10 \%$ \\
- $\quad$ Identifica las normas de circulación vial en el uso de la bicicleta y las normas de seguridad en el & $10 \%$ \\
$\quad$ entorno natural & $10 \%$ \\
- $\quad$ Enumera los recursos materiales necesarios para la práctica del triatlón & $10 \%$ \\
- $\quad$ Realiza un calentamiento específico para una sesión de triatlón/duatlón y/o acuatlón & $10 \%$ \\
- $\quad$ Realiza las acciones básicas del manejo de la bicicleta y las transiciones & $20 \%$ \\
- $\quad$ Participa activamente en las sesiones prácticas independientemente del nivel de destreza alcan- & $20 \%$ \\
$\quad$ zado siempre conociendo las limitaciones de cada uno & \\
- Valora positivamente el triatlón y sus modalidades como medida recreativa de desarrollar y & $10 \%$ \\
$\quad$ mantener la condición física respetando las normas de seguridad en entorno natural y contribu- & yendo a su mantenimiento y conservación \\
\hline
\end{tabular}

Las formas de las que nos valdremos para medir las conductas especificadas en los criterios de calificación son el registro de acontecimientos diarios (diario de sesiones) y una hoja de valoración de una prueba práctica. Se empleará también el examen escrito elaborado a partir de las explicaciones y experiencias prácticas de las diferentes sesiones y los apuntes. La hoja de valoración de la prueba de ejecución práctica es la siguiente: 
Tabla 4. Hoja de evaluación prueba práctica de UD “Nos iniciamos en el triatlón”

VARIABLES A OBSERVAR (1'25 punto/ítem)

Puntos

1. Se coloca el dorsal adecuadamente en los diferentes segmentos

2. Entra y sale de boxes por la zona correcta

3. Se coloca y abrocha el casco adecuadamente

4. Sube correctamente a la bicicleta y en la zona indicada

5. Realiza correctamente los recorridos marcados

6. Baja correctamente de la bicicleta y en la zona indicada

7. Emplea la técnica correcta en la bicicleta

8. Realiza la prueba en el orden correcto

Total:

El examen escrito consiste en la respuesta a una serie de preguntas cortas sobre el mundo del triatlón y sus modalidades y, el medio natural en el que se practica.

\section{4.- REFERENCIAS}

Ascaso Martorell, J., Casterad Seral, J. Generelo Lanaspa, E., Guillén Correas, R. Lapetra Costa, C. Tierz Garcia, M.P., (1996). La actividad física y deportiva extraescolar en los centros educativos. Actividades en la naturaleza. Madrid: MEC.

Decreto n 291/2007, de 14 de septiembre, por el que se establece el currículo de la Educación Secundaria Obligatoria en la Comunidad Autónoma de la Región de Murcia. BORM núm. 221, 27179- 27303.

Granero Gallegos, A. (2007). Una aproximación conceptual y taxonómica a las actividades físicas en el medio natural. Revista Digital Educación Física y Deportes, 107(12). Disponible en http://www.efdeportes.com/

Ley Orgánica 2/2006, de 3 de mayo, de Educación. BOE núm. 106, 17158 -17207.

Machota Blas, V.E. (2012). Propuesta de una unidad didáctica de triatlón en el marco escolar. EmásF, Revista Digital de Educación Física, 19 (4). Disponible en http://emasf.webcindario.com/UD_Triatlon.pdf

Real Decreto 1631/2006, de 29 de diciembre, por el que se establecen las enseñanzas mínimas correspondientes a la Educación Secundaria Obligatoria. BOE núm. 106, 677-773.

Tierra Orta, J. (1996). Actividades recreativas en la naturaleza. En A. Soto Rosales, (coord.), Educación Primaria: actividades en la naturaleza. Huelva: Universidad de Huelva.

Torres, B y Portoles, E (2007). Jóvenes Triatletas. Iniciación al Triatlón. Madrid: Federación Española de Triatlón y Consejería de Deportes Comunidad de Madrid.

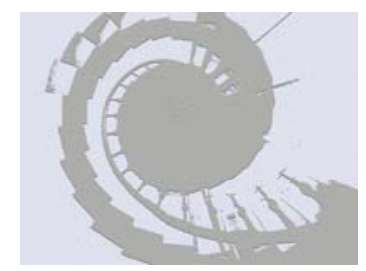

